\title{
Correlation between Optic Disc Damages and Functional Glaucoma Changes
}

\author{
Tomi Petri ${ }^{1}$, Sulejman Zhugli ${ }^{2}$ \\ 1“Ungjillezimi” Clinic, Tirana, Albania \\ ${ }^{2}$ University Hospital Centre "Mother Theresa", Tirana, Albania
}

\begin{abstract}
Glaucoma leads to morphologic changes of the optic nerve head and to functional defects. Morphologic changes in the three-dimensional surface structure of the optic nerve head at its entrance site into the globe can be examined by OCT. The aim of this study is to examine aspects of the correlation between structural and functional changes of the optic nerve head (ONH). This was a prospective study. One hundred six eyes of 53 patients with glaucoma who were followed up during the period from 2013 to 2014 were included at "Ungjillezimi" Clinic in Tirana, Albania. We analysed the data of 106 eyes (53 patients; mean age 65.7 \pm 12.4 years; mean baseline MD)7.1 \pm 5.1). The most common progression parameters were neuroretinal rim change (73\%), followed by $\beta P P A$ enlargement (42\%), DH detection (27\%) and enlargement of a RNFL defect. Structural changes may be more prominent at earlier stages of glaucoma, while functional changes may be more prominent at more advanced disease stages.
\end{abstract}

Keywords: glaucoma, optic nerve, visual field, progression, OCT, evaluation

\section{Introduction}

The optic nerve head, or optic disc, is usually round or slightly oval in shape and contains a central cup. The tissue between the cup and the disc margin is called the neural rim or neuroretinal rim. (1). In normal individuals, the rim has a relatively uniform width and a color that ranges from orange to pink. The size of the physiologic cup is developmentally determined and is related to the size of the disc. For a given number of nerve fibers, the larger the overall disc area, the larger the cup (2). Cup-disc ratio alone is not an adequate assessment of the optic disc for possible glaucomatous damage. Differentiating physiologic or normal cupping from acquired glaucomatous cupping of the optic disc can be difficult. The early changes of glaucomatous optic neuropathy are very subtle. Generalized enlargement of the cup may be the earliest change detected in glaucoma. This enlargement can be difficult to appreciate unless previous photographs or diagrams are available. It is useful to compare one eye with the fellow eye because disc asymmetry is unusual in normal individuals (3-5). The vertical cup-disc ratio is normally between 0.1 and 0.4 , although as many as 5\% of normal individuals will have cup-disc ratios larger than 0.6. Asymmetry of the cup-disc ratio of more than 0.2 occurs in less than $1 \%$ of normal individuals $(6,7)$. This asymmetry may be related to disc size asymmetry. Increased size of the physiologic cup may be a familial trait and it is also seen with high myopia. An oblong insertion of the optic nerve into the globe of individuals with high myopia may also cause a tilted appearance to the optic nerve head. Examination of other family members may clarify whether a large cup is inherited or acquired (8). The aim of this study is to examine aspects of the correlation between structural and functional changes of the optic nerve head $(\mathrm{ONH})$.

\section{Methods}

This was a retrospective study approved by the New York Eye and Ear Infirmary Institutional Review Board and followed the tenets of the Declaration of Helsinki. We included subjects evaluated in the glaucoma referral practice of the authors (JML, RR, CT) in a 10-year period. Details of the study population have been described previously (De Moraes et al. 2011a,b). In short, after an initial visit consisting of a complete ophthalmologic examination, standard achromatic perimetry (24-2 SITA-Standard, Humphrey Field Analyzer II, Carl Zeiss Meditec, Inc., Dublin, CA, USA) and optic disc stereophotographs, patients were re-examined, usually at 3- to 6-month intervals, and the same tests were repeated within 6-12 months.

We selected subjects with glaucomatous optic neuropathy, repeatable VF loss and \$8 SITA VF examinations. All eligible eyes were required to have best corrected visual acuity of $20 / 40$ or better at baseline and a refractive error spherical equivalent $<6$ dioptres. If both eyes of the same patient were eligible, the eye with the greater number of VF tests was enrolled. Eyes with other conditions likely to affect the VF results (retinal, neuro-ophthalmologic, and corneal diseases and significant cataract) were excluded. From this database, we further excluded patients with poor quality stereophotographs within 1 year of the baseline and final SITAVF examinations. Good photograph quality was determined based on even illumination, clarity, centred position of the optic disc and absence of artefacts.

\section{Material and Method}

This was a prospective study. One hundred six eyes of 53 patients with glaucoma who were followed up during the period from 2013 to 2014 were included at "Ungjillezimi" Clinic in Tirana, Albania. In short, after an initial visit consisting of a complete ophthalmologic examination, 


\section{International Journal of Science and Research (IJSR) \\ ISSN (Online): 2319-7064 \\ Index Copernicus Value (2013): 6.14 | Impact Factor (2014): 5.611}

standard achromatic perimetry (24-2 SITA-Standard, Humphrey Field Analyzer II, Carl Zeiss Meditec, Inc., Dublin, CA, USA) and optic disc stereophotographs, patients were re-examined, usually at 3- to 6-month intervals, and the same tests were repeated within 6-12 months. We selected subjects with glaucomatous optic neuropathy, repeatable VF loss and $\geq 8$ SITA VF examinations. All eligible eyes were required to have best corrected visual acuity of 20/40 or better at baseline and a refractive error spherical equivalent Disc photograph review Stereoscopic optic disc photographs taken within 12 months of the baseline and final SITA VF tests were selected for review. If multiple photographs met the criteria, those taken most closely to the baseline and final VF test dates were selected. Stereoscopic optic disc photographs were graded by two glaucoma specialists, masked to their temporal sequence and clinical and VF information, searching for signs of glaucoma progression. Disagreements regarding the occurrence of progression (yes/ no) were adjudicated by a third masked grader. Photographs were graded and progression was determined if at least one of the following four parameters was deemed to be present: (i) neuroretinal rim change; (ii) localized or diffuse widening of RNFL defect; (iii) beta-zone parapapillary atrophy (BPPA) enlargement; and (iv) detection of optic disc haemorrhage (DH). A DH was defined as a splinter-like or flame-shaped haemorrhage on or within the RNFL or neuroretinal rim (910). ВPPA enlargement and detection of $\mathrm{DH}$ were used to define structural endpoints based on reports showing their intrinsic association with glaucoma progression (11). Optic disc progression was defined in cases where there was agreement between two graders and they both described the correct temporal sequence of photographs.

\section{Results and Discussion}

We analysed the data of 106 eyes (53 patients; mean age $65.7 \pm 12.4$ years; mean baseline MD)7.1 \pm 5.1 ). Patients performed a mean of $11.3 \pm 3.1 \mathrm{VF}$ tests spanning $6.5 \pm 1.6$ years. Most patients were diagnosed with primary open angle glaucoma (54\%), followed by exfoliative glaucoma $(15 \%)$, normal-tension glaucoma (13\%), angle-closure glaucoma (12\%) and pigmentary glaucoma (6\%). There was a moderate agreement between the two graders regarding structural progression $(72 \%, \quad \mathrm{kappa}=0.42)$. Following adjudication, 22 eyes $(21.0 \%)$ were determined to have experienced structural progression. One Thirty one eyes (29\%) reached a VF progression endpoint (Fig. 1). The most common progression parameters were neuroretinal rim change $(73 \%)$, followed by ßPPA enlargement $(42 \%), \mathrm{DH}$ detection (27\%) and enlargement of a RNFL defect. The location of the most prominent optic disc topographic change in 53 eyes (56\%). Among these 44 eyes, the most common spatial pattern of progression was diffuse $(66 \%)$, followed by inferior $(18 \%)$ and superior $(16 \%)$. There was no difference in the time intervals between the baseline and final optic disc photographs in cases where the two graders agreed there was progression versus cases in which they disagreed and adjudication was required $(6.8 \pm 1.6$ versus $6.4 \pm 1.7$ years, $\mathrm{p}=0.2$, Mann-Whitney U-test). Eyes with documented optic disc progression had higher mean (15.8 \pm 3.0 versus $15.0 \pm 2.9 \mathrm{mmHg}, \mathrm{p}=0.02)$ and peak $(20.6 \pm 4.6$ versus $19.3 \pm 4.4 \mathrm{mmHg}, \mathrm{p}=0.03$ ) intraocular pressure (IOP) measurements during follow-up than those without progression. There was no difference between groups (progression versus stable) regarding age at baseline assessment $(61.3 \pm 13.7$ versus $63.9 \pm 23.3$ years, $p=0.10)$, central corneal thickness $(\mathrm{CCT})(540.4 \pm 39.4$ versus $539.9 \pm$ 39.5 microns, $\mathrm{p}=0.93)$ or follow-up time $(6.8 \pm 1.5$ versus $6.4 \pm 1.7$ years, $\mathrm{p}=0.10$ ). Eyes with optic disc progression had less negative MD values at baseline than stable eyes () $5.6 \pm$ 4.4 versus) $6.9 \pm 4.9 \mathrm{~dB}, \mathrm{p}=0.03$ ). Eyes with optic disc progression had more rapid rates of VF change than stable eyes $0.66 \pm 0.7$ versus) $0.36 \pm 0.7 \mathrm{~dB} /$ year, $\mathrm{p}<0.01)$ and reached VF PLR endpoints more often $(\mathrm{OR}=1.85$, 95\% CI $=1.10-3.09, \quad \mathrm{p}=0.02$, Table 1), which was independent of age, CCT, baseline MD, follow-up time, and mean and peak IOP values (adjusted OR $=1.78,95 \%$ $\mathrm{CI}=1.04-3.08, \mathrm{p}=0.03$ ).

We investigated the association between structural progression identified with stereoscopic disc photography and SAP progression in a population with treated and established glaucoma seen in a referral practice (12-14). We found that the detection of signs of structural progression during follow-up is associated with an almost twofold increase in the risk of VF deterioration (15). This association was shown to be independent from other covariates and there was moderate spatial consistency between the location of structural change and the hemifield with more rapid decline of VF sensitivity (16). These findings highlight the importance of clinical detection of optic disc progression as a risk factor for visual field loss in glaucoma patients (17). The present study adds new information to the current knowledge of the relationship between structural and functional progression in glaucoma.

\section{Conclusions}

Glaucoma progression can be assessed based on the worsening of functional and/or structural damage. Structural changes may be more prominent at earlier stages of glaucoma, while functional changes may be more prominent at more advanced disease stages. Optic disc assessment is challenging, even in glaucoma diagnosis. Structural changes suggesting glaucoma progression may involve neuroretinal rim shape and width, RNFL and cup-to-disc ratio. The occurrence of disc haemorrhages is not part of the definition for glaucoma progression but has been recognised as a predictive factor for this. In both clinical practice and research, colour photography represents the standard for documentation and monitoring of glaucomatous structural damage. However, the limitations related to the use of optic disc photographs to assess glaucoma progression need to be considered. Based on structural changes of the optic disc, glaucoma progression is mainly qualitatively evaluated.

\section{References}

[1] Zangwill LM, Bowd C, Berry CC, Williams J, Blumenthal EZ, et al. (2001) Discriminating between normal and glaucomatous eyes using the Heidelberg Retina Tomograph, GDx Nerve Fiber Analyzer, and 


\section{International Journal of Science and Research (IJSR) \\ ISSN (Online): 2319-7064 \\ Index Copernicus Value (2013): 6.14 | Impact Factor (2014): 5.611}

Optical Coherence Tomograph. Archives of ophthalmology 119: 985-993. doi: 10.1001/archopht.119.7.985

[2] Reus NJ, de Graaf M, Lemij HG (2007) Accuracy of GDx VCC, HRT I, and clinical assessment of stereoscopic optic nerve head photographs for diagnosing glaucoma. The British journal of ophthalmology 91: 313-318. doi: 10.1136/bjo.2006.096586

[3] Andreou PA, Wickremasinghe SS, Asaria RH, Tay E, Franks WA (2007) A comparison of HRT II and GDx imaging for glaucoma detection in a primary care eye clinic setting. Eye 21: 1050-1055. doi: $10.1038 /$ sj.eye. 6702394

[4] Rudnicka AR, Owen CG, Nightingale CM, Cook DG, Whincup PH (2010) Ethnic differences in the prevalence of myopia and ocular biometry in 10- and 11-year-old children: the Child Heart and Health Study in England (CHASE). Investigative ophthalmology \& visual science 51: 6270-6276. doi: 10.1167/iovs.105528

[5] Shimizu N, Nomura H, Ando F, Niino N, Miyake Y, et al. (2003) Refractive errors and factors associated with myopia in an adult Japanese population. Japanese journal of ophthalmology 47: 6-12. doi: 10.1016/s00215155(02)00620-2

[6] Sawada A, Tomidokoro A, Araie M, Iwase A, Yamamoto T (2008) Refractive errors in an elderly Japanese population: the Tajimi study. Ophthalmology 115: 363-370 e363.

[7] Suzuki Y, Iwase A, Araie M, Yamamoto T, Abe H, et al. (2006) Risk factors for open-angle glaucoma in a Japanese population: the Tajimi Study. Ophthalmology 113: 1613-1617. doi: 10.1016/j.ophtha.2006.03.059

[8] Xu L, Wang Y, Wang S, Jonas JB (2007) High myopia and glaucoma susceptibility the Beijing Eye Study. Ophthalmology 114: 216-220. doi: 10.1016/j.ophtha.2006.06.050

[9] Perera SA, Wong TY, Tay WT, Foster PJ, Saw SM, et al. (2010) Refractive error, axial dimensions, and primary open-angle glaucoma: the Singapore Malay Eye Study. Archives of ophthalmology 128: 900-905. doi: 10.1001/archophthalmol.2010.125

[10] Liang YB, Friedman DS, Zhou Q, Yang X, Sun LP, et al. (2011) Prevalence of Primary Open Angle Glaucoma in a Rural Adult Chinese Population: The Handan Eye Study. Investigative ophthalmology \& visual science 52: 8250-8257. doi: 10.1167/iovs.11-7472

[11] How AC, Tan GS, Chan YH, Wong TT, Seah SK, et al. (2009) Population prevalence of tilted and torted optic discs among an adult Chinese population in Singapore: the Tanjong Pagar Study. Archives of ophthalmology 127: 894-899. doi: 10.1001/archophthalmol.2009.134

[12] Samarawickrama C, Mitchell P, Tong L, Gazzard G, Lim L, et al. (2011) Myopia-related optic disc and retinal changes in adolescent children from singapore. Ophthalmology 118: 2050-2057. doi: 10.1016/j.ophtha.2011.02.040

[13] Tsutsumi T, Tomidokoro A, Saito H, Hashizume A, Iwase A, et al. (2009) Confocal scanning laser ophthalmoscopy in high myopic eyes in a population- based setting. Invest Ophthalmol Vis Sci 50: 52815287. doi: 10.1167/iovs.08-3301

[14]Hwang YH, Yoo C, Kim YY (2011) Myopic Optic Disc Tilt and the Characteristics of Peripapillary Retinal Nerve Fiber Layer Thickness Measured by Spectraldomain Optical Coherence Tomography. Journal of glaucoma.

[15] Hwang YH, Yoo C, Kim YY (2011) Characteristics of Peripapillary Retinal Nerve Fiber Layer Thickness in Eyes With Myopic Optic Disc Tilt and Rotation. Journal of glaucoma.

[16] Abe H, Shirakashi M, Tsutsumi T, Araie M, Tomidokoro A, et al. (2009) Laser scanning tomography of optic discs of the normal Japanese population in a population-based setting. Ophthalmology 116: 223-230. doi: 10.1016/j.ophtha.2008.09.013

[17] Uchida H, Yamamoto T, Araie M, Tomita G, Shirakashi M, et al. (2005) Topographic characteristics of the optic nerve head measured with scanning laser tomography in normal Japanese subjects. Jpn J Ophthalmol 49: 469476. doi: 10.1007/s10384-005-0248-2

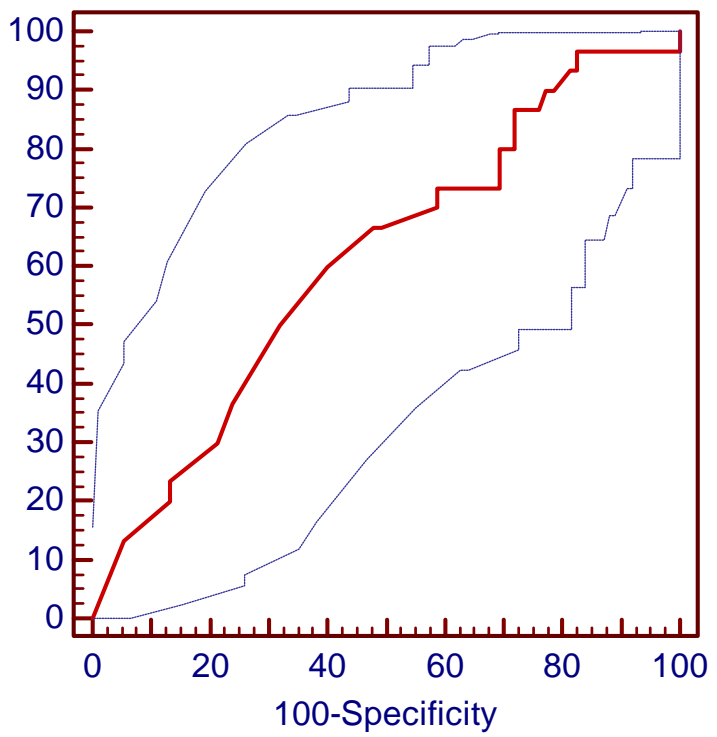

Figure 1: ROC curve for the optic disc progression

Table 1: Global rates of visual field change and number of eyes meeting the predefined trend analysis visual field progression criteria

\begin{tabular}{|c|c|c|}
\hline \multirow{2}{*}{} & \multicolumn{2}{|c|}{$\begin{array}{c}\text { Optic disc progression } \\
\text { evaluation }\end{array}$} \\
\cline { 2 - 3 } & $\begin{array}{c}\text { Progressing } \\
(\mathrm{N}=22)\end{array}$ & $\begin{array}{c}\text { Stable } \\
(\mathrm{N}=84)\end{array}$ \\
\hline $\begin{array}{c}\text { Global rates of visual field change } \\
\text { (dB/year) }\end{array}$ & $0.66 \pm 0.7)$ & $0.36 \pm 0.7$ \\
\hline $\begin{array}{c}\text { Number (\%) of eyes meeting the } \\
\text { visual field progression criteria }\end{array}$ & $9(41.0)$ & $24(28.5)$ \\
\hline
\end{tabular}

